\title{
La fe bahá'í y el aporte al diálogo interreligioso ${ }^{1}$
}

Jenny Pérez G.,

jenny.perez@bahai.cl

Miembro de la Comunidad Bahá'í de Chile

\section{Resumen}

"El bienestar de la humanidad, su paz y seguridad son inalcanzables, a menos que su unidad sea firmemente establecida,"2 es el principio fundamental que mueve a los bahá'ís en el mundo. Bahá'u'lláh, el Fundador de la Fe Bahá'í, dijo que para alcanzar la unidad mundial es necesario que la humanidad reconozca que hay un solo Dios, es decir, que todas las religiones vienen de una misma fuente divina. Otros principios necesarios para lograr la unidad de la humanidad, son la igualdad entre el hombre y la mujer, la eliminación de todas las formas de prejuicio, la armonía entre la ciencia y la religión, la necesidad de la educación para todos y la eliminación de los extremos de riqueza y pobreza.

El diálogo interreligioso crea un ambiente que permite explorar cómo la religión puede ser una fuerza de cohesión, de unidad en la sociedad y como un sistema de conocimiento, que junto con la ciencia, promueve el desarrollo de las civilizaciones. Desde esta perspectiva, la religión a través de las diferentes épocas, ya sea en los tiempos de Krishna, de Moisés, de Jesús, de Buda, de Mahoma o más recientemente de El Báb y Bahá'u'lláh, ha ayudado a la humanidad a refinar su capacidad para alcanzar el bienestar espiritual a la vez que logra el progreso social.

\footnotetext{
${ }^{1}$ Presentación al II Congreso Nacional del fenómeno religioso en el mundo contemporáneo: Mesa Tradiciones religiosas en diálogo. Centro de Estudios Judaicos. Octubre 25 al 27, 2017.

${ }^{2}$ Bahá'u'lláh, Pasajes de los Escritos de Bahá 'u'lláh
} 
Así, la Fe Bahá'í impulsa una visión de diálogo interreligioso que puede llevar a la comprensión de "la raza humana como una sola alma y un solo cuerpo, a la humanidad como una sola familia, y a la humanidad como frutos de un solo árbol y las hojas de una sola rama."3 En este documento se presentan brevemente tres elementos que llevan el concepto de unidad a la práctica: la igualdad entre el hombre y la mujer, la armonía entre ciencia y religión, y la consulta. Se concluye con una corta descripción de un caso en el que se pone en acción el dialogo interreligioso para crear un código de ética, capaz de realzar una cultura de paz y aceptación a la vez que promueve el bien común en nuestra sociedad.

Palabras claves: Bahá'í, religión, Fe Bahá'í, Bahá'u'lláh, dialogo interreligioso

\begin{abstract}
"The well-being of mankind, its peace and security, are unattainable unless and until its unity is firmly established," world. Bahá'u'lláh, the Founder of the Bahá'í Faith, said that to achieve world's unity it is necessary for humanity to recognize that there is only one God, that is, that all religions come from the same divine source. Other important principles to reach unity are equality between men and women, the elimination of all forms of prejudice, harmony between science and religion, the need for education for all and the elimination of the extremes of wealth and poverty.
\end{abstract}

Interreligious dialogue creates an environment that allows exploring how religion can be a force of cohesion, of unity in society and as a system of knowledge, which along with science, promotes the development of civilizations. From this perspective, religion through the different stages of humanity, whether in the times of Krishna, of Moses, of Jesus, of Buddha, of Muhammad or more recently of The Báb and Bahá'u'lláh, has helped to refine the capacity to achieve spiritual well-being while achieving social progress.

\footnotetext{
${ }^{3}$ Bahá'u'lláh, La Epístola al Hijo del Lobo

${ }^{4}$ Bahá'u'lláh, Gleanings from the Writings of Bahá 'u'lláh
} 
Thus, the Bahá'í Faith encourages a vision of interreligious dialogue that can lead to understanding "the human race as one soul and one body, to humanity as one family, and to humanity as the fruits of one tree, and the leaves of one branch." ${ }^{5}$ In this document, the concept of unity is analyzed as the basis for such dialogue and for the contribution of religion in society. In addition, three elements that bring the concept of unity to practice are briefly presented: equality between men and women, harmony between science and religion, and consultation. It concludes with a short description of a case in which interreligious dialogue is put into action to create a code of ethics, capable of enhancing a culture of peace and acceptance while promoting the common good in our society.

Keywords: Bahá'í, religion, Bahá'í Faith, Bahá'u'lláh, interreligious dialogue

\section{La unidad divina}

Dios se ha revelado a lo largo de la historia de la humanidad a través de Mensajeros o Educadores Divinos, fundando cada uno una nueva religión. Así la humanidad ha conocido a Abraham, Krishna, Zoroastro, Moisés, Buda, Jesús y Muhammad. El más reciente de estos Mensajeros es Bahá'u'lláh, quien nació en Persia, lo que hoy en día es Irán, en 1817. Este año celebramos el Bicentenario de Su natalicio.

Cada vez que aparece un Mensajero de Dios se libera en el mundo un nivel más alto de inspiración para la siguiente etapa en el despertar y avance de la humanidad. Un ser humano es llamado a ser portavoz de Dios. Pensamos en Moisés frente a la Zarza Ardiente, en Buda que recibe iluminación debajo del árbol de Bodhi, en el Espíritu Santo que desciende sobre Jesús en forma de una paloma, o en el arcángel Gabriel que se le aparece a Muhammad.

\footnotetext{
${ }^{5}$ Epistle to the Son of the Wolf. http://www.bahai.org/library/authoritativetexts/bahaullah/epistle-son-wolf/ Consultado: 20.10.2017
} 
A mediados del siglo XIX, Dios hizo un llamado a Bahá'u'lláh — cuyo nombre significa la «Gloria de Dios» - para que llevara una nueva Revelación a la humanidad. Durante cuatro décadas fluyeron de Su pluma miles de versos, cartas y libros. En Sus Escritos, esbozó el marco para el desarrollo de una civilización mundial que considera la dimensión espiritual así como la material del desarrollo de la vida humana, trajo enseñanzas espirituales necesarias para reorganizar la sociedad y unificar a todos los pueblos del mundo. Él enseñó que existe un solo Dios, que todas las religiones provienen de un mismo Creador y que ha llegado el tiempo para que la humanidad reconozca que todos somos miembros de una misma familia humana.

Bahá'u'lláh dijo, "Sois los frutos de un solo árbol y las hojas de una sola rama. Trataos unos a otros con extremo amor y armonía, con amistad y compañerismo... Tan potente es la luz de la unidad que puede iluminar toda la tierra." ${ }^{16}$ Con esta visión en mente, la comunidad bahá'í en el mundo hace esfuerzos continuos por promover una conciencia universal sobre la unicidad del género humano. La cual supone una interdependencia orgánica dentro de una entidad social corpórea. Esto admite que el bienestar de las partes integrantes de ese cuerpo está indisolublemente ligado al de su conjunto. Más aún, la unicidad esencial del género humano no se circunscribe a su dimensión física, sino que alcanza a los aspectos sociales y espirituales de la vida humana, nutriendo y desplegando los valores trascendentales del ser humano, considerando la diversidad cultural como expresión de esa verdad básica y universal. Sólo entonces podrán superarse barreras raciales manifiestas. A este respecto, la educación es de capital importancia.

\section{La visión del papel de la religión en la sociedad como un aporte al diálogo interreligioso}

Bahá'u'lláh declaró El bienestar de la humanidad, su paz y seguridad son inalcanzables,

\footnotetext{
${ }^{6}$ Bahá'u'lláh, Pasajes de los Escritos de Bahá 'u'lláh. http://bahailibrary.net/spanish/index.php?option=com content\&view=category\&id=2\&Itemid=9
}

Consultado: 20.10.2017 
a menos que se establezca firmemente su unidad ${ }^{7}$. Estas palabras proféticas, pronunciadas por Bahá'u'lláh durante las décadas finales del siglo diecinueve fueron poco menos que pasadas por alto por los dirigentes de la época. No obstante, en la década final del siglo veinte, la humanidad ha comprendido cada vez mejor su interdependencia y se ha convencido finalmente que ningún individuo, institución o nación puede vivir completamente aislados del conjunto. La crisis del desarrollo y del medio ambiente ha hecho que muchos re-examinen sus opiniones sobre el mundo y a mirar a la tierra como un sistema único, orgánico y unificado. En consecuencia de ello, la búsqueda del equilibrio entre las necesidades de la sociedad y los recursos limitados del mundo natural se está produciendo en el contexto más amplio de la búsqueda del equilibrio, paz, y armonía dentro de la sociedad misma.

El organismo internacional de la Comunidad Bahá'í (Bahá'í International Community) en su declaración sobre El Papel en el Desarrollo Social presentado durante la segunda reunión del Comité Preparatorio para la Cumbre Mundial sobre el Desarrollo Social de Nueva York el 24 de agosto de 1994, describió que:

“'La religión -afirmó Bahá'u'lláh- constituye el medio más potente para el establecimiento del orden y la tranquilidad de cuantos habitan en la Tierra.'

La religión ejerce un influjo poderoso en todas las sociedades. A lo largo de la historia, ha demostrado ser la fuerza principal desencadenante del progreso social, fuerza que motiva a las personas a que desarrollen cualidades espirituales y que hace posible que éstas se sacrifiquen por sus semejantes y por el mejoramiento de sus comunidades.

Los principios espirituales y universales que atesora la religión -la tolerancia, la compasión, el amor, la justicia, la humildad, el sacrificio, la honradez, la unidad y la entrega al bienestar de los demás- son la base de toda civilización progresiva.

\footnotetext{
${ }^{7}$ http://bahai-library.net/spanish/index.php?option=com content\&view=category\&id=2\&Itemid=9 Consultado: 20.10.2017
} 
Al mismo tiempo, también debe reconocerse que a lo largo de todas las épocas la perversión de la religión ha sido la causa fundamental de la desintegración social, la intolerancia, el odio, el sexismo, la pobreza, la opresión y la guerra. Son muchos los problemas actuales, aparentemente insolubles cuya raíz se remonta al mal uso y corrupción de la autoridad religiosa.

Así pues, es evidente que si la religión ha de hacer frente a los múltiples desafíos que la apremian, ella misma debe estar limpia de ignorancia, prejuicios y animosidades.

Tras abandonar toda tendencia a promover una salvación puramente personal o limitada al grupo, la religión debiera recalcar que el bienestar y la realización espiritual de la persona están estrechamente ligados al progreso de la comunidad mundial entera. Los valores de servicio y compromiso activo con la justicia y la unidad hacen que la religión pueda convertirse en una fuerza positiva en el desarrollo social." 8

En uno de sus recientes discursos, Bani Dugal, la representante principal de la Comunidad Bahá'í Internacional ante las Naciones Unidas expresó que:

La comunidad Bahá'i concibe la religión como un sistema evolutivo de conocimiento y práctica, análogo y complementario de la ciencia, dedicado a contribuir al avance de la civilización. En este contexto, una comunidad religiosa se convierte en una comunidad de práctica, comprometida en un proceso sistemático de aprendizaje, ya que se esfuerza por aplicar principios espirituales elevados y ennoblecedores para crear nuevos patrones de vida social. ${ }^{9}$

\footnotetext{
${ }^{8}$ Comunidad Internacional Bahá'í, Declaración sobre el papel de la religión en el desarrollo social https://www.bic.org/statements/el-papel-de-la-religion-en-el-desarrollo-social

${ }^{9}$ Dugal, B. (2017). Women, faith and human rights: Perspectives. New York, Oct. 2, 2017. https://www.bic.org/perspectives/women-faith-and-human-rights Consultado:20.10.2017
} 
Un ejemplo interesante de la aplicación de dos de estos principios espirituales elevados es el de la igualdad entre el hombre y la mujer y la armonía entre ciencia y religión. Ella dice,

"hay que reconocer que ninguno de nosotros, ningún grupo, ni individuo, ni país, ni líder, realmente sabe cómo es una sociedad que realmente se basa en los principios de igualdad de género. Tal sociedad nunca ha existido. Por lo tanto, si bien podemos identificar algunos de los obstáculos que obstaculizan la igualdad de género en nuestro paradigma actual, tenemos que aprender a avanzar hacia un nuevo paradigma donde la igualdad de género sea la norma. El camino a seguir puede no ser del todo claro, pero se necesita urgentemente un camino hacia adelante.

La gran empresa de garantizar los derechos humanos para todas las personas y de lograr la igualdad de género, desde este punto de vista, puede entenderse como un proceso de aprendizaje que se necesita con urgencia. Para desarrollar un nuevo cuerpo de conocimiento, necesitamos recurrir a los grandes sistemas de conocimiento de la humanidad: religión y ciencia. Si bien hay mucho que compartir sobre cada uno, me gustaría ofrecer algunas reflexiones sobre la religión en particular.

La religión llega a las raíces mismas de la motivación humana, permitiéndonos ir más allá de los hechos desnudos y de los fenómenos observables, y ayudándonos a encontrar significado en el universo. La religión nos ayuda a traducir la emoción 
inicial y el entusiasmo por varias causas y programas en un compromiso sostenido a largo plazo con la acción. Después de todo, el proceso de transformación colectiva necesario para lograr la igualdad de género es realmente arduo.

La religión eleva nuestra visión de la naturaleza humana y el carácter humano. No somos solo una amalgama de células y huesos, tejidos y tendones, que de alguna manera tienen el poder emergente de una conciencia superior. La religión nos permite entender que los seres humanos son nobles, que tienen dignidad, y que tienen derecho a ciertos derechos inalienables para salvaguardar su alta posición. A pesar de las identidades secundarias que se usan para categorizarnos según la raza, el género, la afiliación religiosa, el estatus social, etc., nuestra identidad más fundamental como seres humanos debe reformular todas nuestras relaciones a la luz de la unidad. Esta unidad espiritual nos impresiona que nuestro bienestar esté ligado a nuestros vecinos, sin importar su género, religión o asociación política.

Para que la religión funcione junto con la ciencia como un sistema de conocimiento y práctica, también necesitamos desarrollar cualidades científicas y capacidades de investigación y aprendizaje desapasionados. Dado que tenemos que aprender cómo es la verdadera igualdad en la práctica, tenemos que hacer preguntas y formular hipótesis. Necesitamos observar, estudiar y probar rigurosamente las ideas que creemos conducirán al empoderamiento de las mujeres y las niñas. Estas ideas deben medirse en función del estándar de los principios clave como la justicia, la amabilidad, la confiabilidad y la nobleza. Estos esfuerzos deben desarrollarse en un 
patrón de consulta, acción y reflexión para que las observaciones e ideas sobre las prácticas efectivas puedan comprenderse mejor y mejorarse continuamente. En este sentido, entonces, la religión no debería usarse para excusar la osificación de ideales anticuados; debe servir como un sistema de conocimiento dinámico que proporciona mucho del impulso para el progreso y el avance de la humanidad."10

El concepto de La Consulta puede ser vista como un instrumento que puede enriquecer el tema de diálogo interreligioso. El principio de la consulta se asienta en una firme convicción en tomo al poder de la unidad. El hijo de Bahá'u'lláh, 'Abdu'l-Bahá, afirmó que vale la pena esforzarse por alcanzar el acuerdo en cualquier asunto. Él dice que:

"Si coinciden en un asunto, incluso si yerran, es mejor que manifestar desacuerdo y estar en lo cierto, pues esta diferencia derrumba los cimientos divinos. Aunque una de los partes esté en lo cierto, el hecho de disentir será la causa de mil males; pero si las dos partes se ponen de acuerdo, incluso si yerran, será mediante la unidad como la verdad resplandezca y el error se rectifique. ${ }^{11}$

Al presentarse una idea, ésta pasa a ser propiedad de todo el grupo. Aunque simple en apariencia, quizá sea este concepto el más profundo de toda la consulta. De acuerdo con esta regla todas las ideas dejan de ser la propiedad de un individuo, subgrupo o sector de interesados. Cuando este principio es debidamente seguido, se facilita que surjan ideas motivadas por un deseo sincero de servir a los otros, en vez de posibles ideas que emanen de un deseo de engrandecimiento personal o afán de crear escuela.

Para finalizar, quisiera hacer breve mención a un esfuerzo concreto reciente en nuestro ambiente nacional, en el que se han puesto en práctica muchos de los conceptos

${ }^{10}$ Dugal, B. (2017). Women, faith and human rights: Perspectives. New York, Oct. 2, 2017. https://www.bic.org/perspectives/women-faith-and-human-rights. Consultado: 20.10.2017

${ }^{11}$ Los Bahá'ís, publicación de la Comunidad Internacional Bahá'í http://www.bahai.com/losbahais/pag43.htm 
anteriormente descritos. Me refiero a la creación del Código de Ética para el Diálogo en la Convivencia Democrática preparado por el Consejo Asesor Interreligioso, un organismo creado por la Oficina de Asuntos Religiosos del Ministerio Secretaría General de la Presidencia de la República de Chile (ONAR).

Uno de los miembros del Consejo en su presentación en el Segundo Encuentro de Diálogo Interreligioso: “América en Diálogo, Nuestra Casa Común” organizado por el Ministerio de Relaciones Exteriores de Chile a fines de agosto del presente año, describió el trabajo como un esfuerzo en el que "fuimos unos 12 participantes activos, de diferentes orígenes, credos y experiencias. Estuvimos cerca de 18 meses trabajando -desde mediados de 2015 a fines de 2016 - en un ambiente abierto que involucró un grupo de personas cuya relación fue creciendo desde una amable cordialidad hasta una amistad afectuosa.

Puede ser útil destacar el espíritu y algunos de los principios involucrados, en ocasiones de manera explícita y otras veces de forma menos consciente, para alcanzar el objetivo de un emprendimiento de esta naturaleza, el cual representa una profunda reflexión y un constante diálogo que buscó acercar nuestras percepciones sobre un tema central pero no siempre claramente definido.

Posiblemente, el principio de la unidad que ha animado todos nuestros esfuerzos, nutriéndose de la diversidad y riqueza de experiencias de cada participante, haya sido el eje central. Dicha unidad se vio reforzada por una creciente camaradería junto a una sana cuota de alegría.

Un diálogo, que busca establecer un código como el que se generó, implicó un ambiente de consulta franca sobre cada idea compartida, consulta basada en la confianza mutua, la inclusión de cada punto de vista, la colaboración para poder refinar y profundizar cada enunciado, y un significativo grado de humildad y desprendimiento para acercarse a un 
resultado que sentíamos ha de servir a la sociedad que deseamos inspirar e invitar a colaborar en su propio mejoramiento." 12

Del Consejo Interreligioso nació la iniciativa de crear un organismo más permanente para la nación y así poner en práctica el Código de Etica como uno de sus objetivos fundamentales. Esto es ADIR, Asociación Chilena de Diálogo Interreligioso para el Desarrollo Humano. ADIR promueve una cultura de paz y aceptación de nuestros semejantes en la pluralidad de creencias y la promoción del bien común, realizando proyectos de diálogo, encuentro, igualdad y educación.

\section{Referencias}

- Sitio Principal de la Fe Bahá'í: http://www.bahai.org

- Comunidad Internacional Bahá'í (1994). El Papel de la Religión en el Desarrollo Social (Comentarios al borrador de la Declaración y Programa de Acción para el desarrollo social. Presentado por la Comunidad Internacional Bahá'í durante la segunda reunión del Comité Preparatorio para la Cumbre Mundial sobre el Desarrollo Social. New York, 24 August 1994). https://www.bic.org/statements/el-papel-de-la-religion-en-el-desarrollo$\underline{\text { social }}$

- Comunidad Internacional Bahá'í (2017). Toward Prosperity: The Role of Women and Men in Building a Flourishing World Civilization (Commission on the Status of Women Sixty-first session 13 - 24 March 2017).

https://www.bic.org/statements/toward-prosperity-role-women-and-men-buildingflourishing-world-civilization

\footnotetext{
${ }^{12}$ Notas personales de la presentación del Sr. Tiago Masrour en el segundo encuentro de diálogo interreligioso: "América en diálogo, nuestra casa común"

Cuadernos Judaicos ISSN: $07188749 \quad \mathrm{n}^{\circ} 34 \quad$ diciembre 2017 